\title{
IMMUNOTHERAPY VS CHEMOTHERAPY FOR BREAST CANCER: HOW IMPROVED COLLABORATION CAN IMPROVE OUTCOMES
}

\author{
Jasmine Monaghan
}

\section{INTRODUCTION}

Immunotherapy is an emerging treatment option for a range of prominent cancers, including breast cancer. Despite this, there are little to no teachings about immunotherapy as a treatment option for cancers in many undergraduate nursing institutions. This does not provide future health professionals with up-to-date and relevant knowledge for future practice that may involve immunotherapies as their use becomes more commonplace. Immunotherapy describes a treatment modality in which the host immune response to cancer is improved to eliminate cancerous cells better (Couzin-Frankel, 2013). The treatment options for more aggressive forms of breast cancer, including triple negative breast cancer, are limited to traditional treatments such as surgery and chemotherapy (Breast Cancer Foundation NZ, 20 I8a). Immunotherapy represents a new method by which breast cancer can be treated, either as standalone treatment or alongside the traditional options (Bayraktar, Batoo, Okuno \& Gluck, 2019). While the currently approved immunotherapies for breast cancer tend to be experimental, the results to date provide promise that this field may be an option for suffers of breast cancer in the near future. There are many immunotherapeutic treatment options already approved and subsidised for cancers such as melanoma in New Zealand (Cancer Society, 2020). This paper will discuss the potential for immunotherapies to be an alternative treatment for breast cancer as opposed to traditional chemotherapeutic approaches, comparing the benefits and weaknesses for both therapies. Additionally, this paper will highlight, in the form of recommendations, the need for greater collaboration between researchers, New Zealand health professionals and tertiary institutes to allow for improved teaching related to immunotherapies in New Zealand.

\section{BACKGROUND}

Breast cancer is the most prevalent cancer diagnosed in New Zealand women each year, with 3294 diagnoses in 2017 (Ministry of Health, 2019). In breast cancer that is large or fast growing, the recommended treatments are surgery, chemotherapy and hormonal therapy (Breast Cancer Foundation NZ, 2020a). If surgery is not possible to remove a cancerous mass, then treatments such as chemotherapy, radiation and hormonal therapy are suggested in an attempt to reduce tumour size (Breast Cancer Foundation NZ, 2020a). Breast cancer is a heterogenous disease that can be classified into many groups based on a number of characteristics (Zhang, Wang, Yin, Zhang, Zhang and Niu, 2013). One such characteristic is the expression hormone receptors such as progestogen receptor, oestrogen receptor and human epidermal growth factor receptor 2 (Zhang et al., 2013). Of all forms of breast cancer, triple negative breast cancer is the most difficult to treat due to a lack of expression of the aformentioned receptors, therefore treatment with hormone-based therapies is not possible (Zhang et al., 2013).

The current available options for treating triple negative breast cancer in New Zealand are surgery and chemotherapy, and the identification of new treatments for this form of disease is an area of ongoing research (Breast Cancer 
Foundation NZ, 2018a). This ongoing research includes immunotherapies. Promising results have been shown in a clinical trial in which the immunotherapeutic drug Tecentriq was used in conjunction with chemotherapeutic treatment (Schmid et al., 2018). This combination therapy was shown to increase life expectancy of those in the trial by 10 months (Schmid et al., 2018). These results were dependent on the expression of a specific protein, programmed death-ligand I, the protein of which Tecentriq inhibits (Schmid et al., 2018). The following review of relevant literature aims to compare chemotherapy and immunotherapy treatments for breast cancer, with the aim to highlight both similarities to existing treatments as well as the ability of immunotherapies to provide a novel therapeutic alternative. Ultimately, this piece of work aims to promote a conversation around the need for greater collaboration between the researchers and clinicians, including nurses, to further progress the use and understanding of immunotherapies in New Zealand.

\section{DISCUSSION}

\section{Immunotherapy}

Immunotherapy refers to a broad class of therapies in which the host immune system is altered to provide therapeutic benefit, in the case of cancer this involves an improved ability to kill and remove malignant cells (Couzin-Frankel, 2013). These therapies either stimulate or alter a breast cancer patient's immune system in order to recognise and destroy tumour cells successfully (American Cancer Society, 2019a). An important feature of normal immune function is the ability to avoid recognition and destruction of healthy tissues within the body (American Cancer Society, 2019a). One major way in which this is achieved is through 'checkpoint' proteins, which typically function to limit activation of immune cells to keep immune responses controlled (Schmid et al., 2018). The inhibitory nature of these checkpoint proteins is often hijacked by tumour cells, including in breast cancer, to avoid destruction by the immune system (Schmid et al., 20 I8). Immunotherapies have been designed to target the checkpoint proteins and restore the immune response against breast cancer cells (Schmid et al., 20l8). Atezolizumab and Tecentriq are antibody-based therapies that target the programmed death-ligand I protein, a common checkpoint protein found on immune cells and also some tumour cells (Schmid et al., 20 I8). Treatment with the drug leads to the inhibition of programmed death-ligand I function, removing the typical inhibition and ultimately increasing immune function (Schmid et al., 20I8). It has been shown that these therapies have the ability to both reduce the size, and limit the growth, of breast cancer tumours (Schmid et al., 2018). This medication is typically administered intravenously at two week intervals (Schmid et al., 20।8).

\section{Benefits}

Solid tumours, including breast cancer, secrete proteins that inhibit the ability for immune cells to activate and function effectively (Bayraktar et al., 2019) Therefore, to allow for a patient's immune system to target such tumours effectively, it is important to remove potential inhibition (Bayraktar et al., 2019). Through targeting inhibitory checkpoint proteins, such as programmed death-ligand I, it is possible for immunotherapies to restore the natural tumour killing ability of the immune system (Bayraktar et al., 2019). It has been shown that many tumours have the potential to be immunogenic with the correct immune activation (Bayraktar et al., 2019). This is a promising benefit of immunotherapy as although there is a lack of influence on primary tumour growth, the immune system can play an important role in preventing breast cancer metastases (Bayraktar et al., 2019). Immune checkpoint blockade is a form of immunotherapy that has come to light as a promising new clinical approach to advanced forms of breast cancer (Mansour, Teo, Luen, Loi, Teo, \& Luen, 2017).

Checkpoint antibodies were initially tested in treatment of melanoma and renal cell carcinoma, but because of the immunogenic potential of some breast cancer subsets, checkpoint blockade is a promising treatment approach for breast cancer (Mansour et al., 2017). Studies performed in the USA investigated the use of programmed cell death protein I/programmed death-ligand I checkpoint inhibitors in breast cancer patients with all the breast cancer subtypes, including triple negative breast cancer or oestrogen receptor-positive/human epidermal growth factor 
receptor 2-negative breast cancer (Mansour et al., 2017). When administered as a monotherapy, it was shown to be both safe and effective with an overall response rate of between 5 percent and 20 percent, and no adverse effects were seen in majority of patients (Mansour et al., 2017). The durability of response was also noted in these studies, which is a promising indication of having prolonged breast cancer tumour responses to immunotherapy (Mansour et al., 2017). Immunotherapies have been found to be more effective in combination with standard cancer treatments such as chemotherapy (Bayraktar et al., 2019). Data in which both chemotherapy and immunotherapy were used showed that the use of Atezolizumab or pembrolizumab in concert with chemotherapy lead to an improved overall survival rate compared to chemotherapy alone (Bayraktar et al., 2019).

\section{Weaknesses}

Immunotherapy can lead to a number of side effects. These tend to be relatively minor and include irritated eyes, skin rashes of the body and joint pain (Cancer Society, 2020). Less common side effects include headaches, changes of vision, severe abdominal pain and shortness of breath (Cancer Society, 2020).

Checkpoint inhibitors' function is to remove inhibition on the immune system that is typically present during normal immune function (American Cancer Society, 2019a). As such, removal of inhibition has the potential to induce uncontrolled immune cell function and lead to the attack of normal, healthy tissues (American Cancer Society, 2019a). This can affect areas such as the lung, liver and gastrointestinal tract (American Cancer Society, 2019a). The result of this attack on healthy tissue can lead to serious, potentially life-threatening effects (American Cancer Society, 2019a).

Administration of checkpoint inhibitors requires close monitoring of a patient from an appropriate clinician (American Cancer Society, 2019a). When treated rapidly, the majority of immunotherapy related side effects are reversible (American Cancer Society, 2019a). If life threatening complications were to occur, a dose reduction or treatment cessation may be necessary. (García-Aranda \& Redondo, 2019). High doses of corticosteroids are often also used to suppress the immune system if serious over-activation occurs (American Cancer Society, 2019a).

Further research is needed to identify biomarkers capable of predicting a patient's response to immunotherapy and the immune-related adverse effects, allowing for improved patient selection in an attempt to predict adverse effects (García-Aranda \& Redondo, 2019).

\section{Chemotherapy}

Chemotherapy represents the traditional treatment for breast cancer, refering to a broad class of drugs that disrupt the cell cycle, either inhibiting the growth of cancer cells or inducing their death (Breast Cancer Foundation NZ, 2020b). Drugs capable of inhibiting the growth of cancerous cells are referred to as cytostatic, whereas drugs that induce cell death are referred to as cytotoxic (Kummar, Gutierrez, Doroshow, \& Murgo, 2006). Classical chemotherapy tends to be cytotoxic, while newer forms of chemotherapy under development can include cytostatic drugs (Kummar et al., 2006).

Chemotherapy can either be administered prior to surgery, known as neo-adjuvant, or post-surgery, known as adjuvant chemotherapy (Breast Cancer Foundation NZ, 2020b). Adjuvant chemotherapy is administered in an attempt to ensure that microscopic breast cancer cells that are not successfully removed with surgery are killed and all disease is removed (Breast Cancer Foundation NZ, 2020b). Alternatively, neoadjuvant therapy is administered in an attempt to reduce tumour size or lymph node disease prior to surgery, typically with cytotoxic chemotherapy (Kummar et al., 2006). Neo-adjuvant therapy is also used to allow for breast conservation, with a reduction in tumour size prior to surgery possibly removing the need for a full mastectomy (Breast Cancer Foundation NZ, 2020b). Neo-adjuvant chemotherapy can also provide an indication of the likelihood that a tumour will respond to further chemotherapeutic treatment, giving a predictive indicator of better survival (Kim, Osaki, \& Toge, 2005). 
Chemotherapeutic drugs are typically administered intravenously via injection or infusion at an oncology day stay clinic (Breast Cancer Foundation NZ, 2020b). Chemotherapy is usually given in cycles in order to allow for patients to recover from the side effects of the treatment (American Cancer Society, 2019b). The decision of whether an individual is to undergo chemotherapeutic treatment is typically determined by the consideration of a number of key factors (Breast Cancer Foundation NZ, 2020b). These include, but are not limited to, hormone receptor expression (human epidermal growth factor receptor 2), histology and stage of the tumour, presence of high-risk indicators such as lymphovascular invasion, and the age or general health of the patient (Breast Cancer Foundation NZ, 2020b).

\section{Benefits}

It is well established that chemotherapy aids in prolonging the life of sufferers of breast cancer (de Castro Figueiredo Pereira Coelho et al., 2018). Studies have suggested that multiple lines of chemotherapy can lead to an increased overall survival rate in those with advanced breast cancer (Palumbo et al., 2013). Trials conducted in France show that the administration of more than three lines of chemotherapy can induce an overall survival rate benefit of II months (Planchat et al., 20II). Additionally, multiple lines of chemotherapy were shown to reduce metastatic disease complications, decrease the rate of complications such as bone fractures and pleural perfusions, and increase quality of life, compared with those having fewer lines of chemotherapy (Planchat, et al., 20II). It is traditionally less common for elderly women to receive chemotherapeutic treatment; however, this view is changing - in particular in the case of adjuvant chemotherapy (Le Saux et al., 2015). Overall, studies conducted in New Zealand showed that patients receiving more lines of chemotherapy had improved survival rates (Breast Cancer Foundation NZ, 2018b).

\section{Weaknesses}

The major weakness for chemotherapy lies in the range of potential side effects. The nature and severity of these side effects is dependent on the type and dose of medication, as well as treatment length (American Cancer Society, 2019b). Typical side effects include hair loss, mouth sores, weight loss, diarrhoea and vomiting (American Cancer Society, 2019b). Chemotherapy can also negatively impact a patient's immune system by affecting the blood-forming cells in the bone marrow (American Cancer Society, 2019b). Damage to the bone marrow cells increases chances of infection, bruising and fatigue (American Cancer Society, 2019b).The majority of these side effects are short-term and resolve following treatment cessation (American Cancer Society, 20 I9b).

Unfortunately, some side effects induced by chemotherapy are longer lasting. Cognitive dysfunction has been found to be associated with a standard dose of chemotherapy in breast cancer survivors (Wefel, Saleeba, Buzdar, \& Meyers, 20I0). The cognitive decline domains consisted of learning, memory, executive function and processing speed (Wefel et al., 20I0). A study in the USA showed that 21 percent of breast cancer patients receiving chemotherapy evidenced cognitive dysfunction, while 65 percent showed cognitive decline during treatment (Wefel et al., 2010). These results were also seen following extended follow up time, with 61 percent of patients showing cognitive decline (Wefel et al., 20I0). Chemotherapy is also known to cause sexual dysfunction in women following treatment (Usta \& Gokcol, 2017). Commonly used breast cancer chemotherapeutic agents can effect sexuality or sexual function, both directly or indirectly (Usta \& Gokcol, 2017). Indirect damage to a woman's sexuality typically takes the form of reduced sexual drive or feelings of attractiveness, including but not limited to nausea, weight gain and alopecia (Usta \& Gokcol, 2017). Cytotoxic chemotherapy can lead to direct damage to the ovaries, inducing premature menopause (Usta \& Gokcol, 2017). The older a woman is when undergoing chemotherapy treatment, the more likely she is to go through menopause and potentially become infertile as a result (American Cancer Society, 2019b). Chemotherapy has no shortage of devastating side effects; before starting treatment the patient should be advised of the benefits and risks of their situation (Breast Cancer Foundation NZ, 2020b). 


\section{RECOMMENDATIONS}

\section{New Immunotherapy drugs to be available through public system}

Immunotherapies are currently standard treatment for breast cancer patients in many other countries such as the USA, while New Zealand is beginning to follow suit. An immunotherapy medication (trastuzumab emtansine/ Kadcyla) was recently funded in New Zealand for use in breast cancer treatment (Pharmac, 2020a). This hopefully signals the first move for the New Zealand public health system towards increasing immunotherapy availability for breast cancer sufferers. New Zealand lags behind comparable countries in providing access to these new drugs (Breast Cancer Foundation NZ, 2018b). Tecentriq is a drug used in New Zealand for the treatment of lung cancer, although it has proven use in the treatment of breast cancer, it is not funded for this purpose in New Zealand (Pharmac, 2020b). Drugs such as Tecentriq, with proven efficacy in breast cancer, as well as newer, novel therapies need to be assessed faster and allowed funding to treat breast cancer in New Zealand (Breast Cancer Foundation $\mathrm{NZ}, 2018 \mathrm{~b})$.

\section{New Zealand nurses gain a base knowledge on immunotherapies}

There is a fundamental need for New Zealand nurses to have a base knowledge of immunotherapeutic drugs, their use, and possible side effects particularly due to the potentially life threatening nature of some side effects (Wiley et al., 2017). Due to the relatively new nature of immunotherapy, a large portion of the New Zealand nursing force will not have received any concrete training related to immunotherapies during undergraduate studies. Nurses care for people with cancer every day, although they may not be administering the medications, these nurses need to have knowledge of the treatments, allowing for patients to receive reliable information about their condition and treatment (Health and Disability Commissioner, 2020). Accordingly, it is vitally important for more widespread teaching based around immunotherapeutic drugs during undergraduate nursing training, in order to have a well-informed workforce capable of providing the best possible treatment for breast cancer. This will be best achieved through collaboration between immunotherapy researchers, New Zealand health professionals and tertiary institutes providing undergraduate nursing training. This will ensure that all aspects of immunotherapies, ranging from their fundamental principles through to clinical use, can be effectively taught to prospective nurses.

\section{CONCLUSION}

In this paper I have provided a discussion of relevant literature that shows how immunotherapy treatments compare to chemotherapeutic approaches with a focus of breast cancer (which represents one of the major health problems faced by New Zealand women). Traditional treatment of breast cancer involves chemotherapy, which is capable of increasing survival rates of breast cancer patients (Palumbo et al., 2013). These positive outcomes are often coupled with a number of negative side effects that can have life-long implications for breast cancer survivors. Immunotherapy is an emerging form of treatment for a number of cancers of high prevalence in New Zealand, including melanoma, lung cancer and breast cancer (Mansour et al., 2017). Similarly to chemotherapy, the immune system altering nature of immunotherapy can lead to a raft of side effects (García-Aranda \& Redondo, 2019). In this paper, much of the literature suggested that these two treatment modalities are most effective when used collaboratively (Bayraktar, et al., 2019). Despite the promising clinical results seen for many immunotherapies across a range of cancers, this does not translate to proportional teachings in undergraduate healthcare degrees, including nurses. Healthcare professionals must both build and maintain a knowledge of emerging treatment modalities such as immunotherapies to allow for the best possible care to be provided to patients, and therefore the best possible outcomes achieved. New Zealand healthcare needs to come together and collaboratively work with the nursing profession and its undergraduate teaching institutions to help provide up-to-date knowledge about cancer treatment options such as immunotherapy in New Zealand. The utilisation of a collaborative environment in this sense is vital to ensure that graduating health professionals are armed with the best possible knowledge based upon both existing therapeutic options as well as those that are emerging. 
Jasmine Monaghan is a third-year nursing student at the School of Nursing at Otago Polytechnic, Dunedin. Jasmine has a strong interest in breast cancer and emerging treatments such as immunotherapy for this disease.

Correspondence to: Jasmine Monaghan, School of Nursing, Otago Polytechnic | Te Kura Matatini ki Otago, Forth Street, Private Bag 1910, Dunedin 9054, New Zealand. Email: jas.amber@hotmail.com

\section{REFERENCES}

American Cancer Society. (2019a). Immunotherapy for breast cancer. Retrieved from https://www.cancer.org/cancer/breastcancer/treatment/immunotherapy.html

American Cancer Society. (2019b). Chemotherapy for breast cancer. Retrieved from https://www.cancer.org/cancer/breastcancer/treatment/chemotherapy-for-breast-cancer.html

American Society of Clinical Oncology. (2019). Breast cancer: Types of treatment. Retrieved from https://www.cancer.net/cancertypes/breast-cancer/types-treatment

Bayraktar, S., Batoo, S., Okuno, S., \& Glück, S. (2019). Immunotherapy in breast cancer. Journal of Carcinogenesis, 18(2). https:// dx.doi.org/10.4103\%2Fjcar.JCar_2_19

Breast Cancer Foundation NZ. (2018a). Updates in treatments for TNBC. Retrieved from https://www.breastcancerfoundation. org.nz/news-articles/article/updates-in-treatments-for-tnbc?category=blog

Breast Cancer Foundation NZ. (2018b). Insights into living - and dying - with Advanced Breast Cancer in New Zealand. Retrieved from https://breastcancerfoundation.org.nz/lmages/Assets/21893/I/BCFNZ-ABC-Report-20I8.pdf

Breast Cancer Foundation NZ. (2020a). Treatment options. Retrieved from https://www.breastcancerfoundation.org.nz/breastcancer/treatment-options

Breast Cancer Foundation NZ. (2020b). Chemotherapy. Retrieved from https://www.breastcancerfoundation.org.nz/breastcancer/treatment-options/chemotherapy

Cancer Society. (2020). Immunotherapy. Retrieved from https://otago-southland.cancernz.org.nz/cancer-information/treatment/ immunotherapy/?divisionld=20

Couzin-Frankel, J. (2013). Cancer Immunotherapy. Science, 342(6I65), I432-1433. https://doi.org/I0.I I26/science.342.6165.1432

de Castro Figueiredo Pereira Coelho, R., Garcia, S. N., Marcondes, L., da Silva, F. A. J., de Paula, A., \& Kalinke, L. P. (20I8). Impact on the quality of life of women with breast cancer undergoing chemotherapy in public and private care. Investigación y Educación en Enfermería, 36(I), 33-44. https://doi.org/l0.17533/udea.iee.v36nle04

García-Aranda, M., \& Redondo, M. (2019). Immunotherapy: A challenge of breast cancer treatment. Cancers, I/(I2), I822. https:// doi.org/I0.3390/cancers/II21822

Health and Disability Commissioner. (2020). Code of health and disability services consumers' rights. Retrieved from https://www. hdc.org.nz/your-rights/about-the-code/code-of-health-and-disability-services-consumers-rights/

Kim, R., Osaki, A., \& Toge, T. (2005). Current and future roles of neoadjuvant chemotherapy in operable breast cancer. Clinical Breast Cancer, 6(3), 223-232. https://doi.org//0.38I6/CBC.2005.n.024

Kummar, S., Gutierrez, M., Doroshow, J. H., \& Murgo, A. J. (2006). Drug development in oncology: classical cytotoxics and molecularly targeted agents. British Journal of Clinical Pharmacology, 62(I), 15-26. https://doi.org/l0.1/11/j.13652125.2006.02713.x

Le Saux, O., Ripamonti, B., Bruyas, A., Bonin, O., Freyer, G., Bonnefoy, M., \& Falandry, C. (2015). Optimal management of breast cancer in the elderly patient: current perspectives. Clinical Interventions in Aging, 10, 157-174. https://doi.org//0.2147/CIA. S50670

Mansour, M., Teo, Z., Luen, S., Loi, S., Teo, Z. L., \& Luen, S. J. (2017). Advancing Immunotherapy in Metastatic Breast Cancer. Current Treatment Options in Oncology, I8(6), I-15. https://doi.org/I0.1007/s I I864-017-0478-9

Ministry of Health. (2019). Selected cancers 2015, 2016, 2017. Retrieved from https://www.health.govt.nz/publication/selectedcancers-2015-2016-2017

Palumbo, R., Sottotetti, F., Riccardi, A., Teragni, C., Pozzi, E., Quaquarini, E., ... Bernardo, A. (2013). Which patients with metastatic breast cancer benefit from subsequent lines of treatment? An update for clinicians. Therapeutic Advances in Medical Oncology, 5(6), 334-350. https://doi.org/I0.1177//758834013508197 
Pharmac. (2020a). PHARMAC confirms new breast cancer medicine to be funded. Retrieved from https://www.pharmac.govt.nz/ news/2020-03-05-media-release-pharmac-confirms-new-breast-cancer-medicine-to-be-funded/

Pharmac. (2020b). Decision to fund oncology, multiple sclerosis and respiratory treatments. Retrieved from https://www.pharmac. govt.nz/news/notification-2019-10-07-various/

Planchat, E., Abrial, C., Thivat, E., Mouret-Reynier, M.A., Kwiatkowski, F., Pomel, C., ... Durando, X. (20II). Lat lines of treatment benefit survival in metastatic breast cancer in current practice? The Breast, 20(6), 574-578. https://doi.org//0.1016/j. breast.2011.07.010

Schmid, P., Adams, S., Rugo, H. S., Schneeweiss, A., Barrios, C. H., Iwata, H., ... Emens, L. A. (2018). Atezolizumab and NabPaclitaxel in advanced triple-negative breast cancer. The New England Journal of Medicine, 379, 2108-2121. https://doi. org/I0.1056/NEJMoal809615

Usta, O. Y., \& Gokcol, D. (2017). Sexual dysfunction in women with breast cancer Receiving Chemotherapy. International Journal of Caring Sciences, 10(3), 1439-1446.

Wefel, J. S., Saleeba, A. K., Buzdar, A. U., \& Meyers, C. A. (2010). Acute and late onset cognitive dysfunction associated with chemotherapy in women with breast cancer. Cancer Scope, I/6(14), 3307-3523. https://doi.org/l0.1002/cncr.25098

Wiley, K., LeFebvre, K. B., Wall, L., Baldwin-Medsker, A., Nguyen, K., Marsh, L., \& Baniewicz, D. (20I7). Immunotherapy administration: Oncology nursing society recommendations. Clinical Journal of Oncology Nursing, 21, 5-7.

Zhang, J., Wang, Y., Yin, Q., Zhang, W., Zhang, T., \& Niu, Y. (2013). An associated classification of triple negative breast cancer: the risk of relapse and the response to chemotherapy. International Journal of Clinical and Experimental Pathology, 6(7), I380139|. Retrieved from https://www.ncbi.nlm.nih.gov/pmc/articles/PMC3693204/ 